\title{
Inhibiting DNA methylation alleviates cigarette smoke extract-induced dysregulation of Bcl-2 and endothelial apoptosis
}

\author{
Huihui Zeng ${ }^{1,2,3}$, Xianglong Kong ${ }^{4}$, Hongliang Zhang ${ }^{5}$, Yan Chen ${ }^{1,2,3}$, Shan Cai ${ }^{1,2,3}$, Hong Luo ${ }^{1,2,3}$, Ping Chen ${ }^{1,2,3}$
}

\begin{abstract}
INTRODUCTION There is evidence that cigarette smoking participates in disease progression through endothelial apoptosis. Bcl-2 family proteins are essential and critical regulators of apoptosis. We explored whether Bcl-2 plays a role in cigarette smoke extract induced (CSE-induced) endothelial apoptosis. Furthermore, given the involvement of epigenetics in apoptosis and Bcl-2 expression, we hypothesized that CSE-induced apoptosis might be caused by gene methylation.

METHOdS Human umbilical vascular endothelial cells (HUVECs) were treated with CSE, CSE plus 5-aza-2'-deoxycytidine (AZA, an inhibitor of DNA methylation), or AZA and phosphate-buffered saline (PBS). Endothelial apoptosis was determined by Annexin- $\mathrm{V}$ and propidium iodide staining. The expression levels of Bcl-2, Bax, and cytochrome $\mathrm{C}$ ( cyt $\mathrm{C}$ ) were assessed by immunoblotting and RT-PCR. The methylation status of the Bcl-2 promoter was observed by bisulfite sequencing. PCR (BSP).

RESULTS The apoptotic index of endothelial cells in the CSE-treated group increased. Decreased expression of Bcl-2 and high methylation of the Bcl-2 promoter were observed after CSE treatment. AZA alleviated the endothelial apoptosis caused by CSE. AZA treatment also increased Bcl-2 expression along with decreased Bcl-2 promoter methylation.

CONCLUSIONS Inhibiting DNA methylation alleviates CSE-induced endothelial apoptosis and Bcl-2 promoter methylation. Bcl-2 promoter methylation might be involved in CES-induced endothelial apoptosis.
\end{abstract}

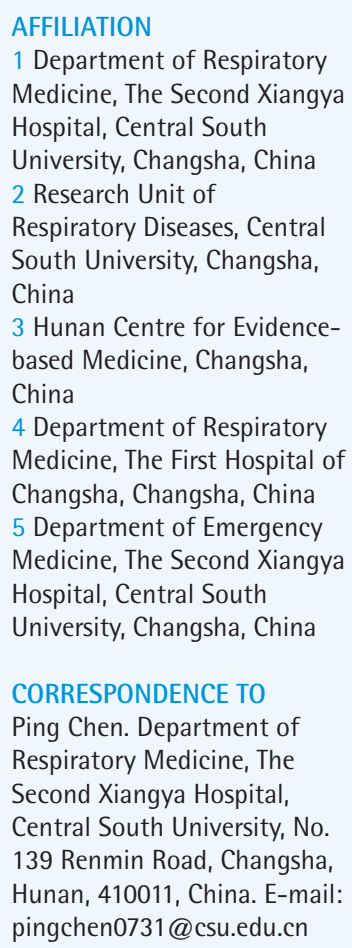

\section{KEYWORDS}

cigarette smoke, endothelial apoptosis, Bcl-2, methylation

Received: 26 October 2019

Revised: 5 February 2020

Accepted: 17 March 2020

\section{INTRODUCTION}

Cigarette smoking is a well-known risk factor for many diseases, such as chronic obstructive pulmonary disease, hypertension, and coronary heart disease, among others. Our previous research found that intraperitoneal injection of cigarette smoke extract (CSE) induced emphysema and injury of the cardiac system in mice ${ }^{1}$. There has been mounting evidence suggesting that cigarette smoking participates in disease progression through endothelial apoptosis ${ }^{2,3}$. It has long been established that cigarette smoke induces endothelial apoptosis ${ }^{4,5}$. However, the underlying mechanisms of the apoptosis process are still poorly understood. Apoptosis is a highly regulated program of 
cell death that can be regulated by Bcl-2 family proteins via mitochondrial maintenance ${ }^{6,7}$. These Bcl-2 family proteins consist of anti- and pro-apoptotic members. Interactions between the classic anti-apoptotic protein B-cell lymphoma-2 (Bcl-2) and the pro-apoptotic protein $\mathrm{Bcl} 2$-associated $\mathrm{X}$ protein (Bax) determine whether the mitochondria will release cytochrome c (cyt C), which is the initial factor of apoptosis ${ }^{6}$. Endothelial mitochondrial maintenance is highly susceptible to cigarette smoking-related damage, and the damage can persist after the cessation of smoking behavior ${ }^{8}$. This result suggests that there is an additional pathogenesis route beyond direct mitochondrial damage.

Some studies have indicated that methylation, an important epigenetic event, participates in the regulation of Bcl-2 and apoptosis ${ }^{9,10}$. Promoter methylation leads to the condensation of chromatin into a compact state, which is inaccessible to transcription factors, causing the downregulation of exon expression. A high methylation status of the Bcl-2 promoter results in reduced expression of Bcl2 mRNA $^{10}$. Recent studies have also demonstrated the involvement of epigenetics in smokers and exsmokers ${ }^{11}$. A previous study from our group showed that hypermethylation of the Bcl-2 promoter took a part in CSE-induced emphysema ${ }^{12}$. Our team also demonstrated that inhibiting DNA methylation might protect endothelial progenitor cells from apoptosis ${ }^{13}$. Taken together, these data present a new possibility that inhibiting DNA methylation might recover the cigarette-induced aberrant methylation of the Bcl-2 promoter and prevent endothelial apoptosis.

Thus, the present study explored the effect of 5-aza2'-deoxycytidine (AZA), inhibiting DNA methylation through DNA methyltransferase enzymes (DNMT), on Bcl-2 methylation status and endothelial apoptosis after treatment with cigarette smoke extract (CSE).

\section{METHODS}

\section{Cell culture}

Human umbilical vascular endothelial cells (HUVECs) were purchased from the American Type Cell Culture Collection (ATCG, lot number: CRL-1730) and were cultured in RPMI-1640 medium (GIBCO, Invitrogen Inc., Carlsbad, CA, USA) containing 10\% heatinactivated foetal bovine serum (GIBCO, Invitrogen Inc.) and $2 \mathrm{mM} \mathrm{L}$-glutamine at $37^{\circ} \mathrm{C}$ in a humidified atmosphere with $5 \% \mathrm{CO}_{2}$.

\section{CSE treatment of HUVECs}

CSE was prepared as previously described ${ }^{4,14}$. Briefly, one unfiltered cigarette (China Tobacco Hunan Industrial CO, Ltd. Tar: $12 \mathrm{mg}$, Nicotine: $1.1 \mathrm{mg}$, Carbon Monoxide: $14 \mathrm{mg}$ ) was burned, and then, the smoke was passed through $25 \mathrm{~mL}$ of phosphatebuffered saline (PBS) using a vacuum pump. This

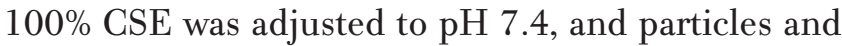
bacteria were removed by filter (Millex-GP syringe filter, Merck Millipore, DE). CSE was prepared fresh for each set of experiments. After our pilot study, this study chose the 5\% CSE concentration to treat cells (Supplementary file). The 100\% CSE was diluted in RPMI-1640 medium to obtain a 5\% CSE medium.

After serum starvation for 24 hours, HUVECs were divided into two groups (CSE and control). The cells in the CSE group were supplemented with 5\% CSE medium for 12 hours. The control group was supplemented with RPMI-1640 medium for 12 hours. During this exposure, the culture medium was replaced every 12 hours to prevent the depletion of essential nutrients. The cells were harvested for the determination of apoptosis and Bcl-2, Bax and cyt $\mathrm{C}$ expression levels.

\section{Inhibiting DNA methylation in cells}

AZA (Sigma, St. Louis, MO, USA), inhibiting DNA methylation through DNMT, was diluted in RPMI1640 medium to obtain $1 \mu \mathrm{M}$ AZA medium. The AZA medium was adjusted to $\mathrm{pH} 7.4$ and filtered through a $0.22 \mu \mathrm{m}$ pore filter (Fisher, $\mathrm{NH}$ ) to remove bacteria and large particles. The AZA medium was prepared fresh before each experiment.

After serum starvation, methylation-inhibited HUVECs were incubated in two groups (AZA+CSE, AZA). The AZA+CSE group was pre-treated with 1 $\mu \mathrm{M}$ AZA medium for 12 hours, followed by incubation with 5\% CSE medium for next 12 hours. The AZA group was incubated with $1 \mu \mathrm{M}$ AZA medium for next 12 hours, then followed by incubation with RPMI1640 medium for 12 hours. During this exposure, the medium was replaced every 12 hours to prevent the depletion of essential nutrients. The cells were harvested for the determination of apoptosis and Bcl2, Bax and cyt $\mathrm{C}$ expression levels.

\section{Apoptosis determination by Annexin-V and propidium iodide staining}

Apoptosis was determined using an Annexin-V FITC 
apoptosis detection kit (Biosea Biotechnology Co., Ltd., Beijing, China) following the manufacturer's instructions. Cells were harvested by centrifugation, resuspended in binding buffer, and successively incubated with $10 \mu \mathrm{L}$ of Annexin-V FITC and $5 \mu \mathrm{L}$ of propidium iodide (PI) stains at room temperature for 15 minutes while protected from light. Apoptosis levels were determined by flow cytometry following a FACSCalibur instrument (BD Biosciences, San Jose, CA, USA) and previous studies ${ }^{15-17}$. Cells that stained negative for PI and positive for Annexin-V were considered to be early apoptotic cells.

\section{Immunoblotting}

HUVECs were homogenized immediately after they were harvested in a buffer containing RIPA lysis and PMSF (Solarbio, Beijing, China), according to the manual. The supernatant was separated after two cycles of centrifugation at $1000 \mathrm{~g}$ for 10 minutes. The isolation of cytosolic fractions was performed following the instructions of the Mitochondria Isolation Kit for Cultured Cells (Pierce, USA). The concentration of both total and cytosolic protein was determined using a bicinchoninic acid (BCA) protein assay (Pierce, USA).

A total of $30 \mu \mathrm{g}$ of protein were separated by SDSPAGE (Beyotime, Beijing, China) and transferred to nitrocellulose (NC) membranes (Millipore, MA, USA). After the protein was transferred, the membranes were blocked with 5\% non-fat milk (dry milk diluted in PBST, PBS containing $0.05 \%$ Tween) for 1 hour at room temperature. After they were blocked, the membranes were washed and incubated overnight with rabbit polyclonal antibodies at $4{ }^{\circ} \mathrm{C}$ (antiBcl-2, Bax and cyt C; 1:1000 dilution; Cell Signaling. Technology, USA). The membranes were washed

\section{Table 1. Primers for RT-PCR}

\begin{tabular}{|c|c|c|}
\hline \multirow[t]{2}{*}{$\mathrm{Bcl}-2$} & forward & 5'-CGCATCAGGAAGGCTAGAGT-3' \\
\hline & reverse & 5'-CAGACATTCGGAGACCACACT-3' \\
\hline \multirow[t]{2}{*}{ Bax } & forward & 5'-AAGCTGAGCGAGTGTCTCAAG-3' \\
\hline & reverse & 5'-CAAAGTAGAAAAGGGCGACAAC-3' \\
\hline \multirow[t]{2}{*}{ cyt C } & forward & 5'-ССССGATACTCTTACACAGC-3' \\
\hline & reverse & 5'-AGTCTGCCCTTCTTCCTTCT-3' \\
\hline \multirow[t]{2}{*}{$\beta$-actin } & forward & 5'-GCACCACACCTTCTACAATGAG-3' \\
\hline & reverse & 5'-GATAGCACAGCCTGGATAGCA-3' \\
\hline
\end{tabular}

four times with PBST and incubated with HRPconjugated goat anti-rabbit IgG (Jackson Immuno Research Laboratories, USA) for 1 hour at room temperature. Bands were detected using an ECL kit (Thermo, USA), and the film was developed and fixed using a developer and fixer kit (Beyotime, Shanghai, China). The expression of each protein was detected and quantitated with Quantity-One software (Bio-Rad Laboratories, CA).

\section{RT-PCR}

Total RNA was extracted from HUVECs as described by Rio et al. ${ }^{18}$. After extraction, the RNA was reversetranscribed using the PrimeScript ${ }^{\circledR}$ RT reagent kit (Takara, Dalian, China) and assayed using SYBR ${ }^{\circledR}$ Premix Ex TaqTM following the manufacturer's instructions. All of the primers were obtained from Sangon Shanghai, China (Table 1). Real-time polymerase chain reaction (PCR) was conducted on the Step-one ABI Real-time RT-PCR system. All mRNA expression levels were calculated relative to that of $\beta$-actin.

\section{Analysis of $\mathrm{Bcl}-2$ promoter methylation status}

The Bcl-2 promoter was determined to exist between $-3000 \mathrm{bp}$ and $+70 \mathrm{bp}$ using the Transcriptional Regulator Element Database (Accession Number 19717, NM 000633). After searching for CpG islands in the UCSC Genome Browser, the CpG islands in the promoter $(-213 \mathrm{bp}$ to $+70 \mathrm{bp})$ were detected and analyzed for their methylation status using bisulfite sequencing PCR (BSP). The primers (Table 2) for BSP were designed using MethPrimer (http://www. urogene.org/methprimer/) and were blasted and confirmed using methBLAST (http://medgen.ugent. be/methBLAST/). A genomic DNA extraction kit (Takara, Dalian, China) was used to extract DNA from cells. Bisulfite conversion of DNA was performed with EpiTect Bisulfate Kits (QIAGEN, Netherlands) following the manufacturer's instructions. After bisulfite modification, nested PCR was performed. The premix solution for the first round contained

\section{Table 2. Primers for BSP}

$\begin{array}{lll}\text { Bcl-2 } & \text { forward } & 5^{\prime} \text {-AGGAATTGGAATAAAAATTITTGTAT-3' } \\ & \text { reverse } & 5^{\prime} \text {-ACAACTTATAATAAATATACTCATCACTA-3' }\end{array}$ 
bisulfite-modified DNA $(2 \mu \mathrm{L})$, Taq $(0.2 \mu \mathrm{L})$ (Takara, Dalian, China), a pair of primers $(20 \mu \mathrm{m}, 1 \mu \mathrm{L}$ each $)$, DEPC-treated H2O (11.8 $\mu \mathrm{L}$; Takara, Dalian, China), and dNTPs (10 mM, $1 \mu \mathrm{L}$; Takara, Dalian, China). PCR cycling was performed as follows: denaturation at $94^{\circ} \mathrm{C}$ for 3 minutes; 30 cycles at $94^{\circ} \mathrm{C}$ for 30 seconds, at $50^{\circ} \mathrm{C}$ for 30 seconds, and at $72^{\circ} \mathrm{C}$ for 1 minute; followed by a final extension at $72^{\circ} \mathrm{C}$ for 5 minutes. For the second round, the primers were changed, and the same PCR cycling parameters were used. After amplification, the PCR products were cloned into the pMD-18T vector (Takara, Dalian, China) and sequenced. The methylation status was calculated by counting the number of methylated $\mathrm{CpGs}$ in all clones, and the methylation status was expressed as the percentage of total CpGs.

\section{Statistical analysis}

A software package (SPSS 16.0; Statistical Product and Service Solutions, USA) was used to perform all statistical analyses. The values are described with means and standard deviation (SD). Student's t-test, one-way ANOVA and Kruskal-Wallis tests were performed to evaluate each group of data and p-values less than 0.05 were considered statistically significant.

\section{RESULTS}

\section{CSE induced apoptosis of HUVECs}

The Annexin V/PI staining showed that the apoptosis index of HUVECs was $23.77 \pm 3.40 \%$ after CSE treatment, compared with $1.57 \pm 0.54 \%$ in the control group (Figure $1 \mathrm{~A}, \mathrm{~B}$, and $\mathrm{C}$ ). As in our previous research ${ }^{4,14}$, the difference between the CSE and control groups was significant $(p<0.05$ by the Student's t-test), supporting the idea that cigarette smoke induces endothelial apoptosis.

\section{CSE regulated $\mathrm{Bcl}-2$, Bax and cytoplasmic cyt C expression in HUVECs}

After a 12-hour incubation in CSE medium, HUVEGs had lower Bcl-2 protein levels $(0.16 \pm 0.05$ vs 0.84 $\pm 0.07 ; \mathrm{p}<0.01$ by the Student's t-test, Figure $1 \mathrm{D}$ and E). Since the balance of anti-apoptotic Bcl2 and pro-apoptotic Bax determines the release of cyt $\mathrm{C}$ to initiate apoptosis, we measured the Bax and cytoplasmic cyt $\mathrm{C}$ levels in the CSE and control groups. Interestingly, Bax protein and cytoplasmic cyt $\mathrm{C}$ protein expression levels were abnormally elevated after CSE treatment (Figure $1 \mathrm{D}$ and $\mathrm{E}$ ). The ratio of $\mathrm{Bcl} 2 / \mathrm{Bax}$ was decreased after CSE treatment, compared with the control group $(0.19 \pm 0.07$ vs 0.78 $\pm 0.07 ; \mathrm{p}<0.01$ by the Student's t-test).

To explore the mechanism by which Bcl-2 protein expression was reduced, the mRNA expression levels of Bcl-2, Bax and cyt $\mathrm{C}$ in the CSE and control groups were detected. As expected, the Bcl-2 mRNA expression was lower in the CSE group than in the

Figure 1. CSE induced apoptosis of HUVECs and regulated protein expression of Bcl-2, Bax and cytoplasmic cyt C
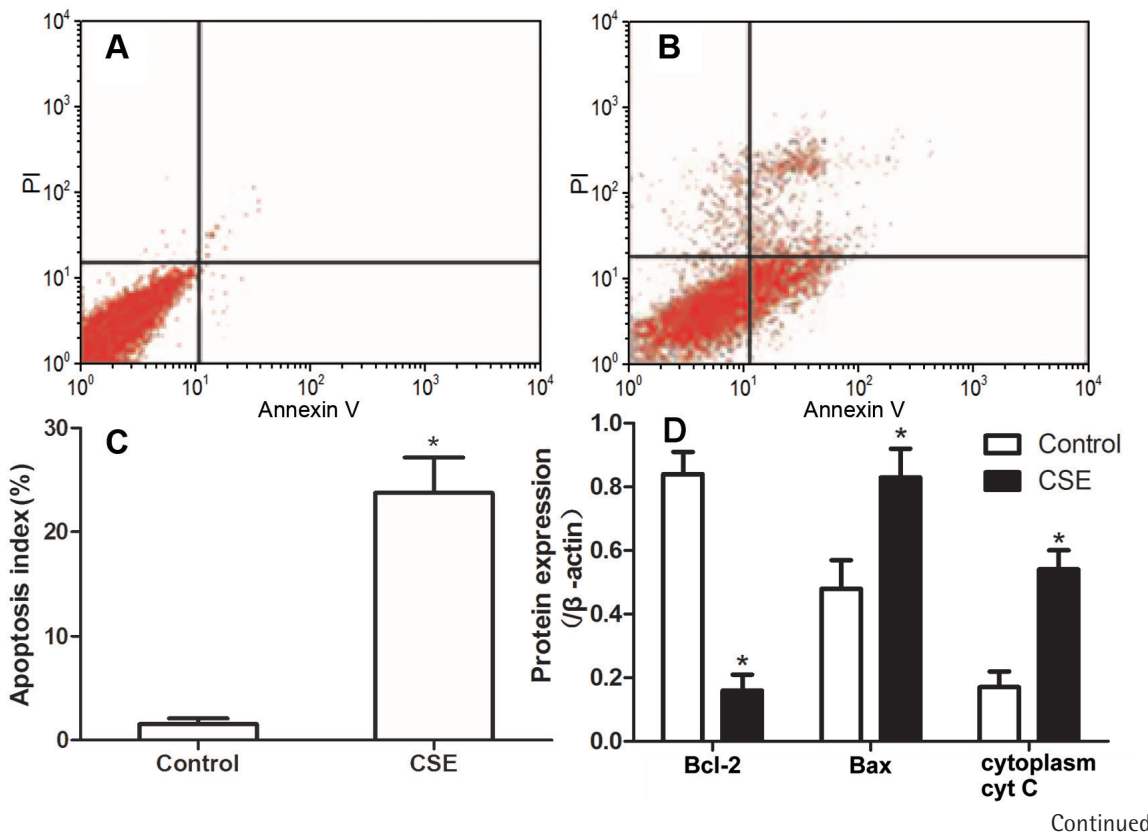
Figure 1. Continued

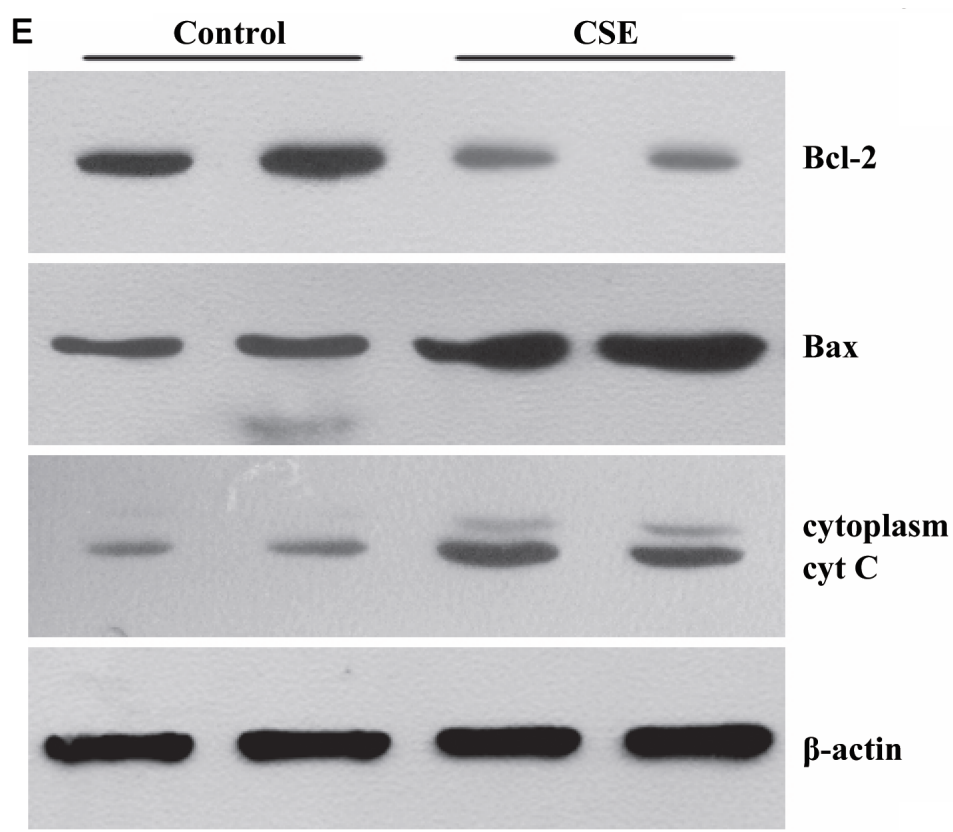

The Annexin V/PI staining detected apoptosis index. A) Flow cytometry result of the control group. B) Flow cytometry result of the CSE group. C) HUVECs apoptosis index was $23.77 \pm 3.40 \%$ after 12 hours' treatment of 5\% CSE, compared with $1.57 \pm 0.54 \%$ in the control group. D) Results from densitometric scan of blotting. Bcl-2 protein from CSE treated HUVECs was detected with decreased expression, compared with the control group ( $0.16 \pm 0.05$ vs $0.84 \pm 0.07 ; p<0.01$ by the Student's t-test). And CSE group also showed increased protein expression of Bax and cytosolic cyt C ( $0.48 \pm 0.09$ vs $0.83 \pm 0.09 ; 0.17 \pm 0.05$ vs $0.54 \pm 0.06$; $p<0.01$ by the Student's t-test). E) Bcl-2, Bax and cytoplasm cyt C proteins were tested through Western blotting in control and CSE groups. *Indicates a statistically significant difference between two groups (p<0.05 by the Student's t-test). Each panel represents $n=5$ independent experiments. The apoptosis index was defined as the percentage of apoptosis cells by flow cytometry. It is shown in the upper right quadrant in Figure $1 \mathrm{~A}$ and $\mathrm{B}$.

control group $\left(0.13 \times 10^{-3} \pm 0.02 \times 10^{-3}\right.$ vs $1.07 \times 10^{-3} \pm$ $0.29 \times 10^{-3} ; \mathrm{p}<0.01$ by the Kruskal-Wallis test, Figure 2 A). However, the mRNA levels of Bax and cyt $\mathrm{C}$ showed no difference between the CSE and control groups (Figure $2 \mathrm{~A}$ ), indicating that the regulation of Bax expression by CSE might be post-transcriptional.

\section{CSE increased the methylation status of the Bcl- 2 promoter}

It is widely accepted that gene transcription rates can regulate mRNA levels, and promoter methylation is an important mechanism of regulation to decrease transcription ${ }^{19}$. Previous studies have demonstrated that gene methylation, especially promoter methylation, participates in the regulation of Bcl-2 expression $^{20,21}$. To investigate whether methylation participates in the Bcl-2 downregulation induced by CSE treatment, we measured methylation status in both the CSE and control groups. BSP showed higher Bcl-2 promoter methylation in the CSE group than in the control group $(1.82 \pm 1.57 \%$ vs $14.55 \pm$ $3.15 \%$; $<0.01$ by the Student's t-test, Figure 2 B).
Therefore, Bcl-2 downregulation might be caused by a high degree of promoter methylation.

\section{Inhibiting DNA methylation restored the CSE-} induced dysregulation of $\mathrm{Bcl}-2$ methylation and expression

Due to the involvement of methylation in GSEtreated HUVECs, we hypothesized that inhibiting DNA methylation would reverse the downregulation induced by CSE. To test this hypothesis, we constructed control, CSE, AZA+CSE and AZA groups. AZA are types of inhibitors frequently used to investigate the methylation process. In this study, HUVECs were treated with AZA and CSE sequentially in the AZA+CSE group and were compared with HUVECs treated with CSE only, HUVECs treated with AZA only, and control HUVECs. BSP analysis showed that the AZA+CSE group was less methylated than the CSE group $(p<0.01$ by the ANOVA test, Figure $2 \mathrm{C}$ ), whereas it had a similar methylation level as the AZA and control groups ( $>>0.1$, Figure $2 \mathrm{C})$. 
Figure 2. Inhibiting DNA methylation restored CSE-induced deregulation of Bcl-2 methylation and mRNA expression
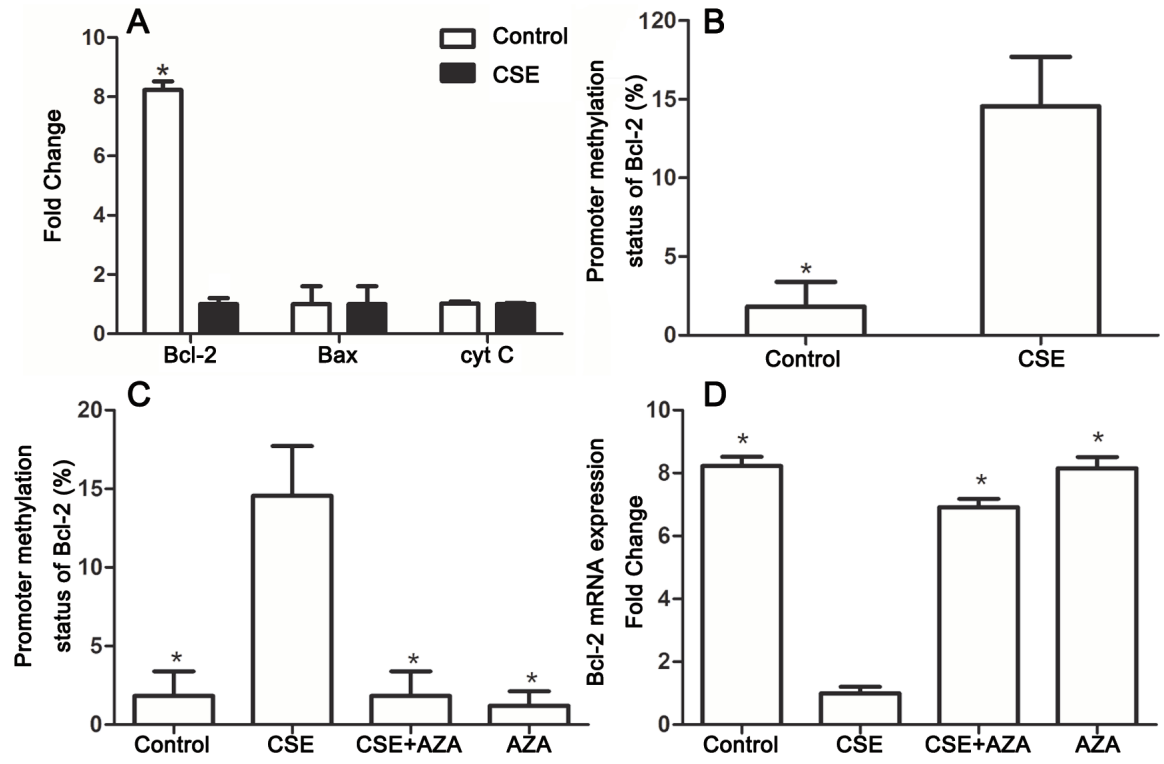

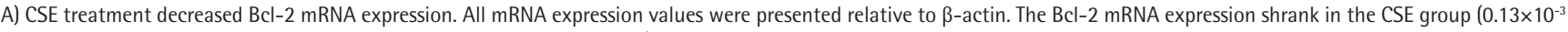
$\pm 0.02 \times 10^{-3}$ vs $1.07 \times 10^{-3} \pm 0.29 \times 10^{-3} ; p<0.01$ by the Kruskal-Wallis test). However, the mRNA expression of Bax and cyt $C$ was found with no significant difference between control and CSE groups. B) CSE increased methylation status of Bcl-2 promoter. The methylation status was presented by counting the number of methylated CpG in all clones and expressing it as the percentage of the whole $\mathrm{CpG}$. The BSP showed higher $\mathrm{Bcl}-2$ promoter methylation in the CSE group than the control $(1.82 \pm 1.57 \%$ vs $14.55 \pm 3.15 \% ; \mathrm{p}<0.01$ by the Student's t-test). C) AZA restored CSE-induced Bcl-2 hypermethylation. BSP detected that the AZA+CSE group showed lower methylation status than the CSE group (1.82 $\pm 1.56 \%$ vs $14.55 \pm 3.15 \% ; p<0.01$ by the ANOVA test), while it presented similar methylation status as AZA and control groups. D) AZA restored CSE-induced downregulation of $\mathrm{Bcl}-2$ mRNA. All mRNA expression values were presented relative to $\beta$-actin. The expression of $\mathrm{Bcl}-2 \mathrm{mRNA}$ was higher in $\mathrm{AZA}+\mathrm{CSE}$ group than $\mathrm{CSE}$ group $\left(0.90 \times 10^{-3} \pm 0.42 \times 10^{-3}\right.$ vs $0.13 \times 10^{-3} \pm 0.02 \times 10^{-3} ; \mathrm{p}<0.01$ by the Kruskal-Wallis test), while AZA+CSE group presented similar Bcl-2 mRNA expression as AZA and control groups. *Indicates a statistically significant difference with CSE group ( $p<0.01$ ). Each panel represents $n=5$ independent experiments in Figure $2 A$ or $n=3$ independent experiments in Figure $2 B$, $C$, and $D$.

As expected, the expression of Bcl-2 mRNA was higher in the AZA+CSE group than in the CSE-only group $\left(0.90 \times 10^{-3} \pm 0.42 \times 10^{-3}\right.$ vs $0.13 \times 10^{-3} \pm 0.02 \times 10^{-3}$; $\mathrm{p}<0.01$ by the Kruskal-Wallis test, Figure 2 D). An analysis of protein levels presented similar results: the AZA+CSE group showed a higher level of Bcl-2 protein expression than the CSE group $(0.16 \pm 0.05$ vs $0.76 \pm 0.05 ; \mathrm{p}<0.01$ by the ANOVA test, Figure 3$)$. All of these results suggest that AZA pretreatment could restore Bcl-2 protein and mRNA expression.

Interestingly, immune blotting showed lower levels of cytosolic cyt $\mathrm{C}$ in the AZA+CSE group than in the CSE group $(0.17 \pm 0.05$ vs $0.54 \pm 0.06 ; \mathrm{p}<0.01$ by the ANOVA test, Figure 3). This finding indicates that the protein expression of cytosolic cyt $\mathrm{C}$ is also reduced by AZA pretreatment. On the other hand, the protein expression of Bax showed no difference in the CSE only and AZA+CSE groups ( $\mathrm{p}>0.1$ by the ANOVA test, Figure 3). Additionally, the protein expression of Bax was the same in the AZA only group and the control group ( $p>0.1$ by the ANOVA test, Figure 3 ). The ratio of $\mathrm{Bcl} 2 / \mathrm{Bax}$ was higher in the AZA+CSE group than in the CSE group $(1.95 \pm 0.07$ vs $0.33 \pm 008 ; \mathrm{p}<0.01$ by the ANOVA test). These results suggest that the regulation of Bax expression might be controlled by a mechanism other than gene methylation.

\section{Inhibiting DNA methylation alleviates CSE- induced endothelial apoptosis}

The flow cytometer detected that the cells in the AZA + CSE group had a lower degree of apoptosis than the cells in the CSE group $(\mathrm{p}<0.01$ by the ANOVA test, Figure 4). Apoptosis in the AZA group was not significantly different than that in the control group ( $p>0.1$ by the ANOVA test, Figure 4 ). It seems that the single use of AZA may not cause more apoptosis than the control treatment. Notably, the abovementioned results demonstrate that inhibiting DNA methylation treatment alleviates CSE-induced endothelial apoptosis. 
Figure 3. Inhibiting of DNA methylation restored CSE-induced downregulation of Bcl-2 protein expression

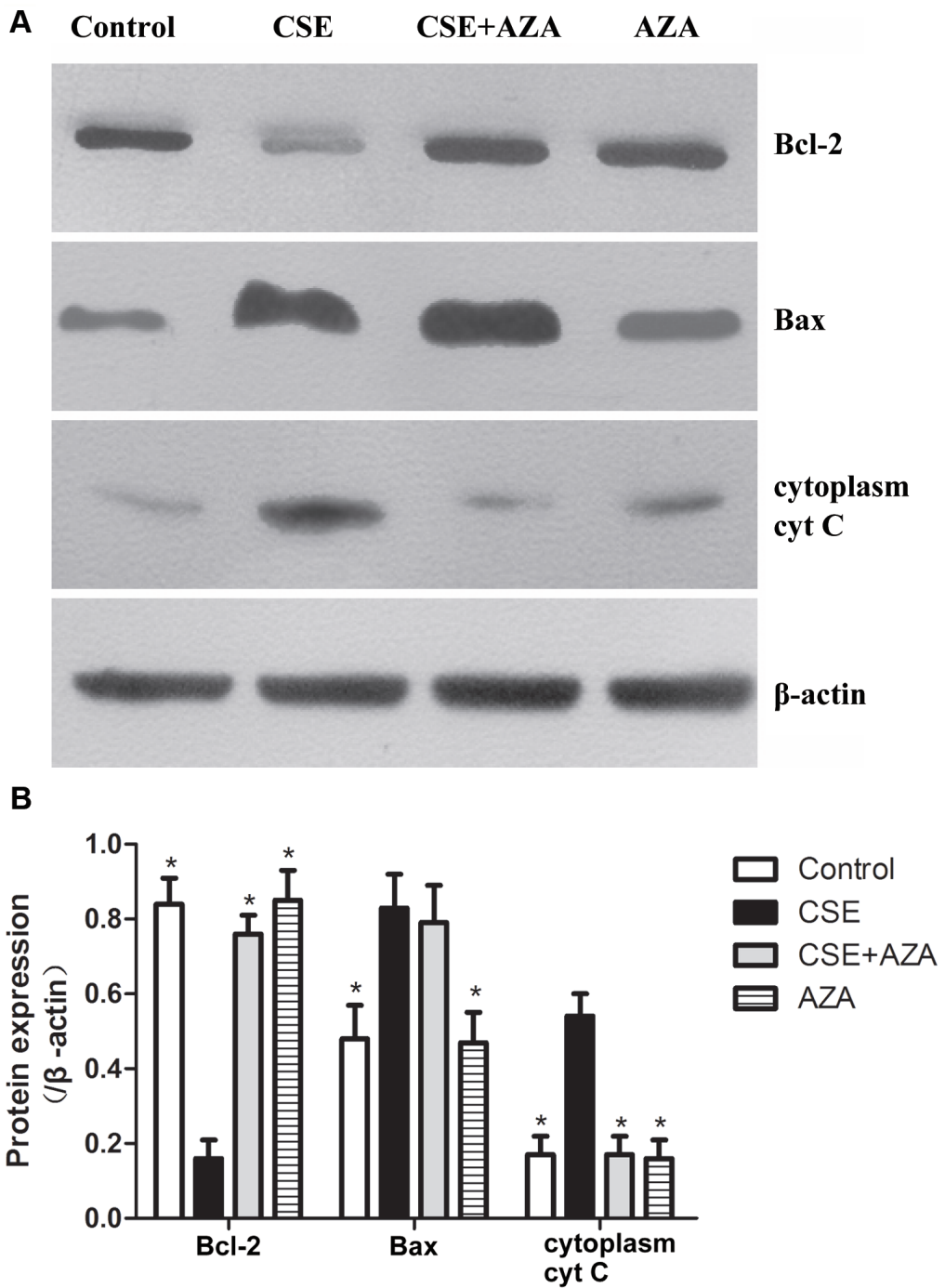

A) $\mathrm{Bcl}-2, \mathrm{Bax}$, and cytoplasm cyt $\mathrm{C}$ protein were tested through Western blotting in control, $\mathrm{CSE}, \mathrm{CSE}+\mathrm{AZA}$, and AZA groups. B) Results from densitometric scan of blotting. AZA+CSE group showed a higher expression of $\mathrm{Bcl}-2$ protein than the CSE group $(0.16 \pm 0.05$ vs $0.76 \pm 0.05 ; \mathrm{p}<0.01$ by the ANOVA test), while it presented similar $\mathrm{Bcl}-2$ protein expression as AZA and control groups. The expression of Bax showed no difference in the single CSE used group and the AZA+CSE group ( $p>0.1$ by the ANOVA test). And the expression of cytosolic cyt $C$ was less in the AZA+CSE group than the CSE group $(0.17 \pm 0.05$ vs $0.54 \pm 0.06 ; p<0.01$ by the ANOVA test). *Indicates a statistically significant difference with the CSE group $(p<0.01)$. Data in Figure 3 B were described with mean \pm SD. Each panel in Figure 3 B represents $n=5$ independent experiments.

Figure 4. Inhibiting of DNA methylation alleviates CSE-induced endothelial apoptosis
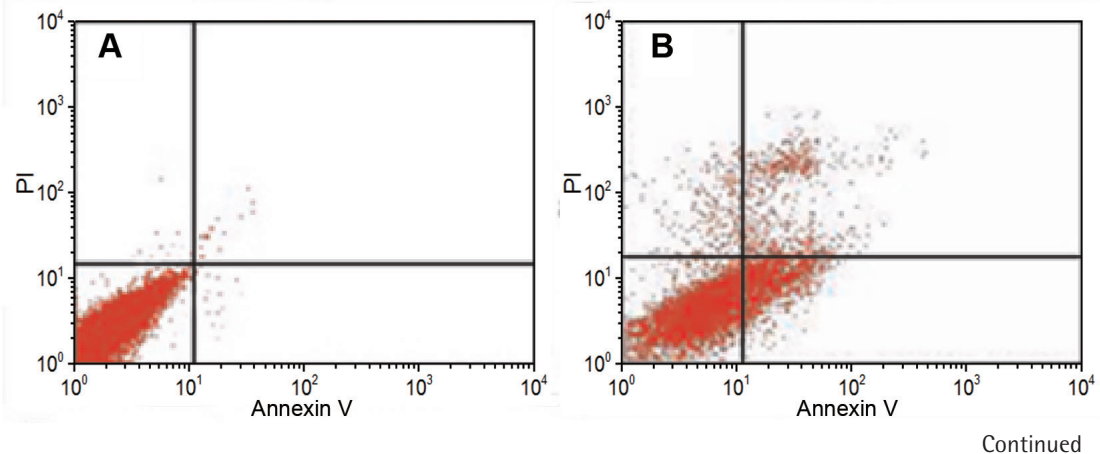
Figure 4. Continued
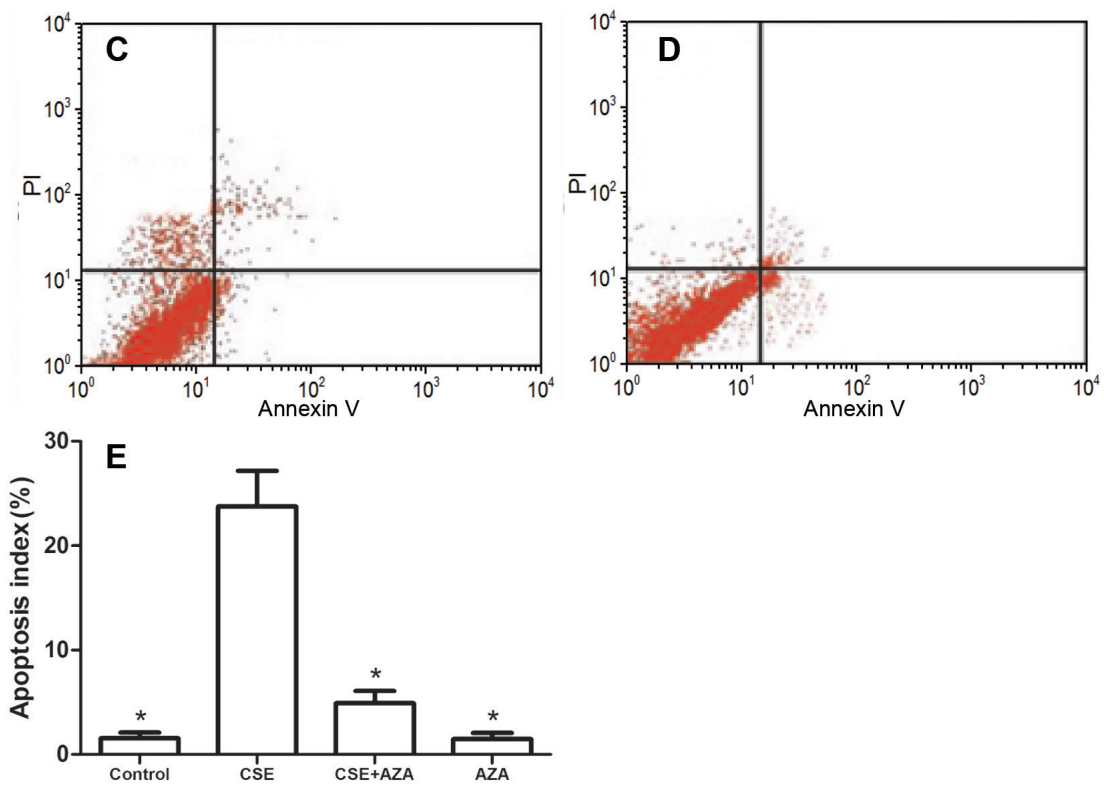

HUVECs were divided into four groups (control, CSE, CSE+AZA and AZA groups). The Annexin V/PI staining detected apoptosis index. A) The HUVECs of control group were supplemented with RPMI-1640 medium for 12 hours. B) Cells in CSE group were supplemented with 5\% CSE medium for 12 hours. C) The AZA+CSE group was pre-treated with 1 $\mu \mathrm{M}$ AZA medium for 12 hours and followed with 5\% CSE incubation for 12 hours. D) The AZA group was incubated with $1 \mu$ M AZA medium for 12 hours. E) The flow cytometer detected that the pretreated AZA led to less apoptosis in AZA+CSE group than in CSE group ( $4.91 \pm 1.17 \%$ vs $23.77 \pm 3.39 \% ; p<0.01$ by the ANOVA test). Compared with the control group, the AZA group did not present a significant difference of apoptosis ( $1.57 \pm 0.54 \%$ vs $1.48 \pm 0.58 \%$; $p>0.01$ by the ANOVA test). *Indicates a statistically significant difference with the CSE group $(p<0.01)$. Data in Figure $4 \mathrm{E}$ were described with mean \pm SD. Each panel in Figure 4 E represents $n=3$ independent experiments.

\section{DISCUSSION}

Cigarette smoke is inhaled into the lungs and diffuses into the alveoli and blood. The first barrier is the mucus on the surface of the airways and alveoli. The cigarette smoke inhaled into lungs diffuses and dissolves into mucus. The mucus is a kind of liquid, and CSE could mimic this liquid phase better than cigarette smoke. Notably, cigarette smoke is a risk factor for many diseases, such as coronary artery disease, hypertension, rheumatoid arthritis and chronic obstructive pulmonary disease (COPD), among others ${ }^{20,22,23}$. Our group also found injury of the cardiac and respiratory system in CSE-induced emphysema models ${ }^{1}$. All of the abovementioned studies indicate that cigarette smoke could induce multisystem damage, not only to the pulmonary system. The endothelia cells might be a direct target for cigarettes, and HUVECs are probably the most accepted models for human endothelial cells ${ }^{24-26}$ and are widely used in endothelial research ${ }^{27,28}$. This study found that CSE induced the apoptosis of HUVECs, which is consistent with results of previous studies ${ }^{4,13,14,29}$. Furthermore, this study found Bcl-2 hypermethylation after CSE incubation, and inhibiting methylation could reverse the apoptosis and hypermethylation. Prior studies have documented that endothelial apoptosis plays a role in different diseases of different systems $\mathrm{s}^{30-33}$. It is possible that cigarette smoke might cause multisystem diseases, or at least endothelium-associated diseases, through endothelial apoptosis.

Mechanistically, it is widely accepted that Bcl-2 family proteins regulate apoptosis through the release of cyt $\mathrm{C}$ from the mitochondria. Bcl-2, a principal antiapoptotic protein, is an agonist of the pro-apoptotic protein Bax. It has been demonstrated that decreased Bcl-2/Bax causes the release of cytosolic cyt C from mitochondria, eventually triggering apoptosis ${ }^{34}$. As in previous studies ${ }^{35,36}$, our group found CSE treatment decreased the expression of $\mathrm{Bcl}-2$ and the $\mathrm{Bcl}-2 / \mathrm{Bax}$ ratio and increased cytosolic cyt $\mathrm{C}$ protein levels. The imbalance between Bcl-2 and Bax coincided with increased endothelial apoptosis. These results suggest that CSE might induce endothelial apoptosis by reducing Bcl-2 expression, resulting in the release of cytosolic cyt $\mathrm{C}$.

We found that the downregulation of Bcl-2 protein levels was consistent with mRNA levels and gene 
methylation status. The results also showed that CSE incubation led to higher methylation of the Bcl-2 promoter. Furthermore, inhibiting DNA methylation restored CSE-induced hypermethylation of the Bcl-2 promoter and mRNA expression. CpG methylation, in which methyl groups are attached to cytosine bases next to guanine ( $\mathrm{CpG}$ site), is an emerging and important pre-transcriptional regulation mechanism ${ }^{37}$. Promoter methylation inhibits transcription factor binding and target gene transcription, which blocks gene expression. According to the Hoffman and $\mathrm{Hu}^{21}$ research of the $\mathrm{CpG}$ island, this work detected an important promoter element of human Bcl-2 located within P2. Hypermethylated P2 will turn off and silence Bcl-2 expression ${ }^{21}$. Our results suggest that CSE might lower Bcl-2 expression by increasing the methylation status of the Bcl-2 promoter.

In addition, we demonstrated that the AZA alleviated CSE-induced endothelial apoptosis by restoring Bcl-2 methylation status. It might be deduced that CSE-induced hypermethylation of the Bcl-2 promoter participates in endothelial apoptosis, even in endothelium-associated diseases. Since DNA methylation patterns are mostly not heritable, they can result from many factors, such as the environment, food, and stress. The DNA methylation status can be different in populations with the same genetic background. The abovementioned deduction can explain why only certain smokers suffer cigarette-induced diseases within genetically related populations, while others with the same genetic background do not develop diseases. Once DNA methylation develops, it will stably propagate during a lifetime, leading to a persistent downregulation of gene expression. The propagation of DNA methylation can also explain why some cigarette-induced diseases, for example COPD, continue progressing after smoke cessation. However, AZA treatment demethylates many genes, not just Bcl-2. To clarify whether the hypermethylation of the Bcl-2 promoter directly induces endothelial apoptosis, additional studies to specifically target the methylation and demethylation of the Bcl-2 promoter are required. The CRISPR system precisely and efficiently mutates genes through sgRNA and $\mathrm{Cas}^{38}$. Exogenous plasmids can also mutate genes in precise loci. Both gene editing methods can identify the exact hypermethylated loci and demonstrate the effect of promoter methylation.
In a future study, the CRISPR system might be an appropriate method to clarify the function of Bcl-2 methylation.

The protein levels of Bax, the proapoptotic member of the Bcl-2 family, were increased after CSE incubation in this study. AZA did not reverse the pattern of Bax expression, and Bax mRNA levels showed no difference between the CSE and control groups. The results suggest that the CSE-induced high expression of Bax might be regulated after transcription or even translation, but further study is required.

\section{CONCLUSIONS}

This study presents the novel result that inhibiting DNA methylation can alleviate CSE-induced endothelial apoptosis and Bcl-2 promoter methylation. Furthermore, Bcl-2 promoter methylation might be involved in CSE-induced endothelial apoptosis.

\section{REFERENCES}

1. Zhang Y, Cao J, Chen Y, et al. Intraperitoneal injection of cigarette smoke extract induced emphysema, and injury of cardiac and skeletal muscles in BALB/C mice. Exp Lung Res. 2013;39:18-31. doi:10.3109/01902148.2012.745910

2. Lockhart PB, Bolger AF, Papapanou PN, et al. Periodontal disease and atherosclerotic vascular disease: does the evidence support an independent association? A scientific statement from the American Heart Association. Circulation. 2012;125:2520-2544. doi:10.1161/cir.0b013e31825719f3

3. Chen Y, Dawes PT, Mattey DL. Polymorphism in the vascular endothelial growth factor A (VEGFA) gene is associated with serum VEGF-A level and disease activity in rheumatoid arthritis: differential effect of cigarette smoking. Cytokine. 2012;58:390-397. doi:10.1016/j.cyto.2012.02.018

4. Yang M, Chen P, Peng H, et al. Cigarette smoke extract induces aberrant cytochrome-c oxidase subunit II methylation and apoptosis in human umbilical vascular endothelial cells. Am J Physiol Cell Physiol. 2015;308:C378-C384. doi:10.1152/ajpcell.00197.2014

5. Brown MB, Hunt WR, Noe JE, et al. Loss of cystic fibrosis transmembrane conductance regulator impairs lung endothelial cell barrier function and increases susceptibility to microvascular damage from cigarette smoke. Pulm Circ. 2014;4:260-268. doi:10.1086/675989

6. Youle RJ, Strasser A. The BCL-2 protein family: opposing activities that mediate cell death. Nat Rev Mol Cell Biol. 2008;9:47-59. doi:10.1038/nrm2308

7. Tabas I, Ron D. Integrating the mechanisms of apoptosis induced by endoplasmic reticulum stress. Nat Cell Biol. 2011;13:184-190. doi:10.1038/ncb0311-184

8. Masayesva BG, Mambo E, Taylor RJ, et al. Mitochondrial 
DNA content increase in response to cigarette smoking. Cancer Epidemiol Biomarkers Prev. 2006;15:19-24. doi:10.1158/1055-9965.epi-05-0210

9. Nishioka C, Ikezoe T, Yang J, Udaka K, Yokoyama A. Simultaneous inhibition of DNA methyltransferase and histone deacetylase induces p53-independent apoptosis via down-regulation of Mcl-1 in acute myelogenous leukemia cells. Leuk Res. 2011;35:932-939. doi:10.1016/j.leukres.2011.04.004

10. Vinci S, Giannarini G, Selli C, et al. Quantitative methylation analysis of BCL2, hTERT, and DAPK promoters in urine sediment for the detection of nonmuscle-invasive urothelial carcinoma of the bladder: a prospective, two-center validation study. Urol Oncol. 2011;29:150-156. doi:10.1016/j.urolonc.2009.01.003

11. Wan ES, Qiu W, Baccarelli A, et al. Cigarette smoking behaviors and time since quitting are associated with differential DNA methylation across the human genome. Hum Mol Genet. 2012;21:3073-3082. doi:10.1093/hmg/dds135

12. Zeng H, Shi Z, Kong X, et al. Involvement of B-cell CLL/ lymphoma 2 promoter methylation in cigarette smoke extract-induced emphysema. Exp Biol Med (Maywood). 2016;241:808-816. doi:10.1177/1535370216635759

13. He ZH, Chen P, Chen Y, et al. Dual effects of cigarette smoke extract on proliferation of endothelial progenitor cells and the protective effect of 5-aza-2'-deoxycytidine on EPCs against the damage caused by CSE. Biomed Res Int. 2014;2014. doi:10.1155/2014/640752

14. Chen Y, Hanaoka M, Chen P, Droma Y, Voelkel NF, Kubo K. Protective effect of beraprost sodium, a stable prostacyclin analog, in the development of cigarette smoke extract-induced emphysema. Am J Physiol Lung Cell Mol Physiol. 2009;296:L648-L656. doi:10.1152/ajplung.90270.2008

15. Jalilzadeh N, Samadi N, Salehi R, et al. Novel nanovehicle for delivery and efficiency of anticancer auraptene against colon cancer cells. Sci Rep. 2020;10:1606. doi:10.1038/s41598-020-58527-0

16. Capdevielle C, Desplat A, Charpentier J, et al. HDAC inhibition induces expression of scaffolding proteins critical for tumor progression in pediatric glioma: focus on EBP50 and IRSp53. Neuro Oncol. 2019. doi:10.1093/neuonc/noz215

17. Poon IK, Parkes MA, Jiang L, et al. Moving beyond size and phosphatidylserine exposure: evidence for a diversity of apoptotic cell-derived extracellular vesicles in vitro. J Extracell Vesicles. 2019;8:1608786. doi:10.1080/20013 078.2019.1608786

18. Rio DC, Ares M Jr, Hannon GJ, Nilsen TW. Purification of RNA using TRIzol (TRI reagent). Cold Spring Harb Protoc. 2010;2010:pdb.prot5439. doi:10.1101/pdb.prot5439

19. Blaze J, Roth TL. Evidence from clinical and animal model studies of the long-term and transgenerational impact of stress on DNA methylation. Semin Cell Dev Biol. 2015. doi:10.1016/j.semcdb.2015.04.004
20. Zeng H, Kong X, Peng H, et al. Apoptosis and Bcl-2 family proteins, taken to chronic obstructive pulmonary disease. Eur Rev Med Pharmacol Sci. 2012;16(6):711-727. PMID: 22913201

21. Hoffman AR, Hu JF. Directing DNA methylation to inhibit gene expression. Cell Mol Neurobiol. 2006;26:425-438. doi:10.1007/s10571-006-9057-5

22. Wu CH, Lin HH, Yan FP, Wu CH, Wang CJ. Immunohistochemical detection of apoptotic proteins, p53/Bax and JNK/FasL cascade, in the lung of rats exposed to cigarette smoke. Arch Toxicol. 2006;80:328336. doi:10.1007/s00204-005-0050-4

23. Rahman MM, Laher I. Structural and functional alteration of blood vessels caused by cigarette smoking: an overview of molecular mechanisms. Curr Vasc Pharmacol. 2007;5:276-292. doi:10.2174/157016107782023406

24. Guilpain P, Mouthon L. Antiendothelial cells autoantibodies in vasculitis-associated systemic diseases. Clin Rev Allergy Immunol. 2008;35:59-65. doi:10.1007/s12016-007-8069-3

25. Carnevale R, Raparelli V, Nocella C, et al. Gut-derived endotoxin stimulates factor VIII secretion from endothelial cells. Implications for hypercoagulability in cirrhosis. J Hepatol. 2017;67:950-956. doi:10.1016/j.jhep.2017.07.002

26. Thornton C, Al-Rashed F, Calay D, et al. Methotrexatemediated activation of an AMPK-CREB-dependent pathway: a novel mechanism for vascular protection in chronic systemic inflammation. Ann Rheum Dis. 2016;75:439-448. doi:10.1136/annrheumdis-2014-206305

27. Ende G, Poitz DM, Wiedemann E, et al. TNF-alphamediated adhesion of monocytes to endothelial cells-The role of ephrinA1. J Mol Cell Cardiol. 2014;77:125-135. doi:10.1016/j.yjmcc.2014.10.010

28. Adela R, Nethi SK, Bagul PK, et al. Hyperglycaemia enhances nitric oxide production in diabetes: a study from South Indian patients. PloS One. 2015;10:e0125270. doi:10.1371/journal.pone.0125270

29. Gong J, Zhao H, Liu T, et al. Cigarette smoke reduces fatty acid catabolism, leading to apoptosis in lung endothelial cells: implication for pathogenesis of COPD. Front Pharmacol. 2019;10:941. doi:10.3389/fphar.2019.00941

30. Hong J, Kim K, Kim JH, Park Y. The Role of Endoplasmic Reticulum Stress in Cardiovascular Disease and Exercise. Int J Vasc Med. 2017;2017. doi:10.1155/2017/2049217

31. Cocchiaro P, De Pasquale V, Della Morte R, et al. The multifaceted role of the lysosomal protease cathepsins in kidney disease. Front Cell Dev Biol. 2017;5:114. doi:10.3389/fcell.2017.00114

32. Thenappan T, Ormiston ML, Ryan JJ, Archer SL. Pulmonary arterial hypertension: pathogenesis and clinical management. BMJ. 2018;360. doi:10.1136/bmj.j5492

33. Makwana O, Flockton H, Smith GA, Watters GP, Nisar R, Fields W. Mechanisms of whole smoke conditioned media induced cytotoxicity to human aortic endothelial cells. Toxicol In Vitro. 2019;58:239-244. 
doi:10.1016/j.tiv.2019.03.011

34. Wang C, Youle RJ. The role of mitochondria in apoptosis*. Annu Rev Genet. 2009;43:95-118. doi:10.1146/annurevgenet-102108-134850

35. Kawashima A, Koide K, Ventura W, et al. Effects of maternal smoking on the placental expression of genes related to angiogenesis and apoptosis during the first trimester. Plos One. 2014;9:e106140. doi:10.1371/journal.pone.0106140

36. Sun Y, An N, Li J, et al. miRNA-206 regulates human pulmonary microvascular endothelial cell apoptosis via targeting in chronic obstructive pulmonary disease. J Cell Biochem. 2019;120:6223-6236. doi:10.1002/jcb.27910

37. Bergman Y, Cedar H. DNA methylation dynamics in health and disease. Nat Struct Mol Biol. 2013;20:274281. doi: $10.1038 / \mathrm{nsmb} .2518$

38. Mei Y, Wang Y, Chen H, Sun Z, Ju X. Recent Progress in CRISPR/Cas9 Technology. J Genet Genomics. 2016;43:63-75. doi:10.1016/j.jgg.2016.01.001
ACKNOWLEDGEMENTS

This study was supported by National Key Clinical Specialist Construction Programs of China; National Natural Science Foundation of China 81370143, 81170036, 81400032; Natural Science Foundation of Hunan Province 09JJ3036, 2019JJ50877; and Hunan Medical Research Plan Foundation B2014-145.

\section{CONFLICTS OF INTEREST}

The authors have completed and submitted the ICMJE Form for Disclosure of Potential Conflicts of Interest and none was reported.

FUNDING

National Natural Science Foundation of China Award Number: 81370143, Recipient: Ping Chen. National Natural Science Foundation of China Award Number: 81170036, Recipient: Yan Chen. National Natural Science Foundation of China Award Number: 81400032 , Recipient: Huihui Zeng. Natural Science Foundation of Hunan Province Award Number: 09JJ3036, Recipient: Xianglong Kong; Award Number: 2019JJ50877, Recipient: Huihui Zeng. Hunan Medical Research Plan Foundation Award Number: B2014-145, Recipient: Xianglong Kong.

PROVENANCE AND PEER REVIEW

Not commissioned; externally peer reviewed. 